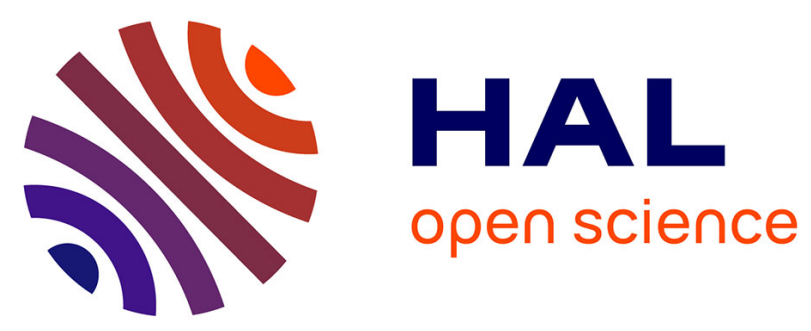

\title{
Le management stratégique des réseaux inter-organisationnels à l'épreuve des comportements opportunistes: élaboration d'un cadre d'analyse
}

François Fulconis, Gilles Paché

\section{- To cite this version:}

François Fulconis, Gilles Paché. Le management stratégique des réseaux inter-organisationnels à l'épreuve des comportements opportunistes: élaboration d'un cadre d'analyse. La Revue des Sciences de Gestion, 2008, N 230, Mars-Avril,, pp. 35-43. 10.3917/rsg.230.0035 . hal-02304739

\section{HAL Id: hal-02304739 \\ https://hal-amu.archives-ouvertes.fr/hal-02304739}

Submitted on 3 Oct 2019

HAL is a multi-disciplinary open access archive for the deposit and dissemination of scientific research documents, whether they are published or not. The documents may come from teaching and research institutions in France or abroad, or from public or private research centers.
L'archive ouverte pluridisciplinaire HAL, est destinée au dépôt et à la diffusion de documents scientifiques de niveau recherche, publiés ou non, émanant des établissements d'enseignement et de recherche français ou étrangers, des laboratoires publics ou privés. 


\title{
Le management stratégique des réseaux inter-organisationnels à l'épreuve des comportements opportunistes Élaboration d'un cadre d'analyse
}

\author{
par François Fulconis et Gilles Paché
}

François Fulconis

Maître de Conférences à l'Université d'Avignon et des Pays de Vaucluse

IUT d'Avignon

Site Agroparc - BP 1207

337 chemin des Meinajariés

84911 AVIGNON CEDEX

Téléphone : 04.90.84.14.00

Télécopie : 04.90.84.38.15

E-mail : francois.fulconis@ univ-avignon.fr

\section{Gilles Paché}

Professeur à l'Université Montpellier I

Centre de Recherche sur le Transport et la Logistique

413, Avenue Gaston Berger

13625 AIX-EN-PROVENCE CEDEX 1

Téléphone : 04.42.93.90.33

Télécopie : 04.42.26.17.32

E-mail : gpache@univ-montp1.f 


\title{
Le management stratégique des réseaux inter-organisationnels à l'épreuve des comportements opportunistes Élaboration d'un cadre d'analyse
}

\author{
par François Fulconis et Gilles Paché
}

François Fulconis

Maître de Conférences à l'Université d'Avignon et des Pays de Vaucluse

Gilles Paché

Professeur à l'Université Montpellier I

\section{Résumé}

Le management stratégique des réseaux inter-organisationnels à l'épreuve des comportements opportunistes : élaboration d'un cadre d'analyse

Par François Fulconis et Gilles Paché

Les relations entre entreprises sont l'objet de nombreux travaux. Ils s'intéressent tout particulièrement à l'amélioration de la performance de ces relations à partir de la mise en œuvre de stratégies coopératives au sein de réseaux inter-organisationnels (RIO). Une certaine orthodoxie de la recherche en théorie des organisations et en management stratégique présente les comportements opportunistes sous un jour négatif et largement contre-productif pour les relations entre membres des RIO. Seul un engagement durable et une totale confiance en l'autre partie seraient ainsi capables de créer de la valeur. L'article remet en cause cette conception traditionnelle en indiquant qu'un opportunisme «modéré » pourrait au final avoir des impacts positifs insoupçonnés sur le fonctionnement des RIO.

Mots-clés : Management stratégique, Opportunisme, Réseaux inter-organisationnels, Théorie des organisations. 


\begin{abstract}
The strategic management of inter-organizational networks faced with opportunism: an elaboration of the framework

By François Fulconis and Gilles Paché

Many academic works have been devoted to the study of the relations between firms, especially with the improvement of these relations starting with the implementation of cooperative strategies within inter-organisational networks (ION). Mainstream research in organisational theory, and strategic management presents opportunistic behaviour in a negative light and is largely counter-productive for relationships between ION members. Only long-term involvement and total trust in the partners would create value. The article questions this traditional view by indicating that a more "moderate" opportunism could at last have an unsuspected positive impact on the functioning of ION.
\end{abstract}

Key words: Inter-organizational networks, Opportunism, Organization theory, Strategic management.

\title{
Resumen
}

La dirección estratégica de las redes interorganizacionales cuestionada por los comportamientos oportunistas : elaboración de un marco de análisis.

Por François Fulconis y Gilles Paché

Las relaciones entre empresas dar lugar a muchas investigaciones. Estas se focalizan en particular en la mejoría de la efectividad de estas relaciones a partir de la implementación de estrategias de cooperación en el marco de redes interorganizacionales (RIO). Cierta ortodoxia de la investigación en materia de teoría de organizaciones y en gestión estratégica presenta los comportamientos oportunistas como negativos y claramente contra-productivos para las relaciones entre miembros de las RIOs. Así, sólo un compromiso duradero y una confianza total en la otra parte serían capaces de crear valor. El artículo cuestiona este enfoque tradicional al indicar que un oportunismo "moderado" podría finalmente tener insospechados impactos positivos sobre el funcionamiento de las RIOs.

Palabras clave: Gestión estratégica, Oportunismo, Redes interorganizacionales, Teoría de organizaciones. 


\section{Le management stratégique des réseaux inter-organisationnels à l'épreuve des comportements opportunistes Élaboration d'un cadre d'analyse}

La radicale remise en question des politiques d'intégration verticale qui ont structuré le capitalisme de la première moitié $\mathrm{du} \mathrm{XX}^{\mathrm{e}}$ siècle, apparaît comme une réalité peu contestable aux observateurs avertis. Le modèle de management de la multinationale sucrière Tate and Lyle, contrôlant directement l'ensemble d'une filière agro-industrielle, des plantations jusqu'aux points de vente, en passant par les moyens de production et de transport (P. Chalmin, 1983), appartient à une histoire lointaine où la possession d'actifs matériels et immatériels semblait être, pour les équipes dirigeantes, la seule stratégie possible en vue de maîtriser les turbulences de l'environnement. Pour les entreprises d'aujourd'hui, l'alternative est plutôt de savoir mobiliser un «portefeuille » de compétences extérieures et, grâce à elles, concevoir, fabriquer et commercialiser des produits dans les meilleures conditions de coût, de qualité et de délai (M. Capraro et G. Baglin, 2002). En suivant B. de Montmorillon (1997), nul doute que nous sommes entrés dans une ère nouvelle, celle de la croissance contractuelle, fondée sur une multiplicité de transactions entre entreprises juridiquement et financièrement autonomes, mais organisationnellement de plus en plus interdépendantes, tendance d'ailleurs confirmée par de nombreuses recherches académiques insistants sur le développement de relations contractuelles complexes entre entreprises (C. Ménard, 2004 ; B. Baudry, 2006).

Depuis le début des années 1980, les travaux académiques portant sur la nature et l'intensité des relations inter-organisationnelles sont d'une extrême richesse, quels que soient d'ailleurs leurs points d'entrée: démarches coopératives de tous types (alliances, partenariats d'impartition ou symbiotiques), nouvelles formes d'organisation, chaînes logistiques étendues, développement des systèmes d'information inter-organisationnels (SIIO) ou, plus 
largement, management de systèmes d'offre multi-acteurs. L'objectif n'est pas de synthétiser cette abondante littérature et de rendre compte des différentes controverses qui l'ont traversée et la traversent encore. Il s'agit davantage de s'interroger sur un point qui prête largement à débat: que se passe-t-il lorsque, au sein de réseaux inter-organisationnels (RIO), des partenaires impliqués dans une relation d'échange ne se montrent pas systématiquement loyaux dans leurs comportements ? Par RIO, nous entendons ici un système d'échange entre entreprises, aux ressources et compétences complémentaires, développant un projet commun dans le cadre de stratégies coopératives ; par nature, leurs relations sont à la fois durables et étroites, condition indispensable pour mener à bien le projet qui les unit (G. Paché et C. Paraponaris, 2006).

La réponse traditionnelle apportée par la théorie des organisations et le management stratégique est bien connue: en situation d'opportunisme, latent ou révélé, la relation d'échange risque de s'interrompre à plus ou moins brève échéance, faute d'une confiance suffisante entre les co-contractants (P. Milgrom et J. Roberts, 1997 ; B. Quélin, 2002). Ce caractère «destructif »s'explique aisément puisque si un partenaire apparaît peu (voire pas) fiable, il sera difficile d'envisager avec lui la conduite d'un projet commun de longue haleine. En effet, il sera impossible de prédire s'il profitera ou ne profitera pas d'une occasion donnée pour agir dans son propre intérêt en enfreignant les règles établies au sein du RIO et en fragilisant de ce fait les relations entre partenaires (O. Williamson, 1975). Mieux vaut dès lors éviter de s'engager durablement, et si une relation d'affaires est ponctuellement nouée avec lui, il sera indispensable de surveiller le plus étroitement possible ses actions.

C'est ce thème que nous souhaitons « revisiter » en cherchant à savoir si un opportunisme modéré de la part de preneurs de décisions ne peut pas avoir, au contraire, un impact positif sur la qualité et les effets attendus de la coopération. Une telle position est iconoclaste, notamment par rapport aux travaux de recherche d'obédience néo-institutionnelle. Elle a pour ambition à la fois de rompre avec une vision dichotomique (et à visée normative) opposant le triptyque confiance-coopération-efficience au triptyque opportunisme-compétitioninefficience, et de réhabiliter les attitudes et comportements opportunistes pour éclairer d'un jour nouveau le fonctionnement effectif des organisations. L'opportunisme apparaît comme une réalité institutionnelle qui réclame de la part des co-contractants une vigilance certaine, 
leur évitant ainsi de tomber dans le piège d'une sorte de «sclérose organisationnelle » et de routines bureaucratiques. Plus encore, l'opportunisme modéré d'un acteur peut être bénéfique pour lui et pour l'acteur qui le subit : l'opportuniste enfreint certes des clauses contractuelles en usant de tromperie, par exemple en ne respectant pas un accord d'exclusivité conclu avec un partenaire, mais il va peut-être permettre à ce dernier de profiter en retour de l'expérience acquise dans la mise en œuvre des relations d'échange avec d'autres entreprises. Ce point de vue hétérodoxe sera défendu à partir d'une lecture critique de travaux majeurs sur le thème.

\section{L'opportunisme : clarification conceptuelle préalable}

Concept-clé de l'approche néo-institutionnelle, l'opportunisme se situe au cœur de nombreuses études conduites sur la dynamique des relations inter-organisationnelles, notamment dans le cadre des canaux de distribution (R. Stump et J. Heide, 1996 ; R. Achrol et G. Gundlach, 1999 ; A. Joshi et R. Stump, 1999 ; J. Brown et al., 2000 ; K. Wathne et J. Heide, 2000), mais aussi, de manière beaucoup plus large, dans le cadre de l'économie des contrats (O. Hart et B. Holmstrom, 1987 ; P. Milgrom et J. Roberts, 1997). Les recherches conduites condamnent vigoureusement les comportements opportunistes dans la mesure où ils sont censés contrarier la mise en œuvre de stratégies coopératives interentreprises. Ceci explique que, selon une approche «orthodoxe», les réflexions se soient principalement portées sur la manière d'éradiquer voire d'empêcher l'émergence de l'opportunisme.

Mais de quoi parle-t-on exactement lorsqu'il est question de comportements opportunistes, latents ou révélés, entre agents économiques ? Le sens donné par le courant néo-institutionnel est sans ambiguïté, et n'a rien à voir avec la confusion parfois entretenue entre «être opportuniste» et «saisir des opportunités ». En effet, des entreprises industrielles et commerciales peuvent tout à fait s'engager mutuellement dans des coopérations durables en n'hésitant pas, si l'occasion se présente, à développer de façon ponctuelle des relations d'affaires avec d'autres entreprises, pourtant écartées au départ du projet collaboratif. Il ne s'agit pas ici d'opportunisme, mais d'un simple recours au marché, d'ailleurs parfois prévu par des contrats de coopération autorisant une mise en concurrence des membres du RIO avec 
des entreprises extérieures. Une clarification conceptuelle s'avère donc nécessaire pour éviter toute erreur d'interprétation.

\subsection{Un comportement « déviant» mais rationnel et volontaire}

Selon O. Williamson (1985, p. 47), l'opportunisme peut être défini comme «la recherche de l'intérêt personnel avec ruse. Ceci inclut, sans être exhaustif, les formes les plus flagrantes telles que le mensonge, le vol et la tromperie (...). Plus généralement, l'opportunisme se réfère à la divulgation d'une information tronquée ou déformée, à tout effort calculé pour induire en erreur, désinformer, déguiser, obscurcir, autrement dit rendre confus ». De ce point de vue, si l'opportunisme apparaît comme un comportement «déviant», il n'en demeure pas moins totalement rationnel dans la mesure où il résulte d'un calcul : dans telle ou telle situation, j' aurai plus à gagner à agir de manière opportuniste qu'à coopérer et être loyal avec autrui. Il n'est donc pas systématiquement présent (ou révélé) dans des relations d'échange, néanmoins il apparaît comme un penchant naturel qui pousse les agents économiques à rechercher en priorité leur propre intérêt, quitte à tricher ou à trahir leur parole. Cette recherche, précisent K. Wathne et J. Heide (2000), peut d'ailleurs être effectuée de manière passive ou active :

- un agent économique fait preuve d'opportunisme passif, ou par omission, lorsqu'il tait à son ou ses partenaire(s) une information conditionnant le devenir de leur(s) relation(s) comme, par exemple, sa faible adaptabilité ;

- en revanche, un agent économique fait preuve d'opportunisme actif s'il ment volontairement ou s'il présente de façon biaisée certains faits ou certaines données le concernant.

On pourrait penser, en première approximation, que les comportements opportunistes s'apparentent à un espace discrétionnaire dont profitent les agents économiques pour jouer avec les règles $d u$ jeu, ainsi que nous l'apprend l'analyse stratégique initiée par M. Crozier et E. Friedberg (1977). Une telle vision paraît erronée car la transgression des règles du jeu par un acteur résulterait dans ce cas d'une tactique ou d'une politique personnelle pour accroître son influence, mais sans chercher à nuire directement à autrui. Or, au contraire, un 
comportement opportuniste sous-entend qu'il peut y avoir volontairement manipulation de l'autre partie prenante à la transaction pour entraver son développement (P. Joffre, 1999). Si une telle manipulation est possible, c'est que la rationalité limitée des preneurs de décisions ne leur permet pas de percevoir automatiquement son existence et ses impacts néfastes. Notons toutefois que si une entreprise ne respecte pas ses engagements vis-à-vis de ses partenaires, elle ne fait pas nécessairement preuve d'opportunisme ; c'est le cas lorsque ses agissements apparemment déloyaux résultent d'une mauvaise connaissance des projets des partenaires en situation d'asymétrie informationnelle (C. Jameux, 2004).

\section{2. « Être opportuniste » ou « saisir des opportunités »?}

Dans un souci de clarté, il convient de souligner qu'adopter ou tenter d'adopter des comportements opportunistes ne doit pas être confondu avec les moyens mis en œuvre par une entreprise pour «saisir des opportunités » en dehors du RIO auquel elle appartient. Dans ce cas, son action ne s'accompagne d'aucun effort calculé ou d'aucune volonté visant à abuser les autres membres du réseau. Ladite entreprise profite de ce que l'environnement externe lui permet de réaliser en termes d'actions tactiques pour accroître sa part de marché par exemple, ou tout simplement se pérenniser. Ce point a d'ailleurs été souligné très tôt par P.-Y. Barreyre (1968) dans son analyse des obstacles psychologiques à une politique d'impartition réussie. Par un abus de langage dommageable, force est d'admettre que certains travaux ont confondu ensuite le fait de «saisir des opportunités» et le fait « d'être opportuniste ». Un exemple concret permet pourtant de distinguer clairement les deux postures.

Imaginons qu'un distributeur A et un industriel signent un accord de coopération pour que, lors de chaque lancement d'un nouveau produit, soient engagées des opérations publipromotionnelles communes. Il n'y aura pas opportunisme si l'industriel saisit à un moment donné l'opportunité de conclure avec un distributeur B un accord traditionnel de référencement. On comprend d'ailleurs aisément qu'en élargissant son portefeuille de clients, l'industriel cherche à réduire sa dépendance potentielle vis-à-vis du distributeur $\mathrm{A}$ et qu'il 
veuille conserver, dans le respect des règles établies, son autonomie décisionnelle ${ }^{1}$. En revanche, il y aura par exemple opportunisme si, pour faciliter son référencement, l'industriel s'engage à livrer au distributeur B des informations stratégiques et confidentielles sur le distributeur A, en trompant délibérément la confiance de ce dernier. Risque d'ailleurs clairement identifié par P.-A. Julien et al. (2003, p. 14) lorsqu'ils écrivent, en introduction d'un ouvrage collectif sur l'entreprise-réseau : «Pour que le réseau fonctionne bien, il faut que les membres échangent de l'information dont une partie est de nature stratégique; cette information peut inciter quelques membres du réseau à se retirer pour en profiter seuls, ou encore à se retrouver chez des concurrents ».

Le problème de fond n'est donc pas que l'un des partenaires du projet commun cherche à développer de nouvelles relations économiques et à assurer sa pérennité en s'ouvrant sur son environnement; les travaux conduits de longue date par M. Granovetter (1973), et plus récemment par B. Uzzi (1997), dans le cadre de RIO ont souligné la pertinence et la légitimité managériale de cette démarche. L'essentiel de l'analyse consiste à savoir si une telle ouverture s'effectue de façon volontaire en enfreignant ou non les règles (les clauses contractuelles) gouvernant les relations partenariales. Dans ce cas de transgression, et uniquement dans ce cas, il est possible de parler de comportement opportuniste au sens de O. Williamson (1975, 1985, 1993), et non pas de simple opportunité d'affaires saisie à un moment donné, sans chercher à manipuler ou à tromper l'autre partie.

\section{L'approche « orthodoxe» ou comment se prémunir des comportements opportunistes}

La lecture qu'il est possible de faire de l'opportunisme par l'approche orthodoxe d'obédience néo-institutionnelle est une condamnation sans équivoque compte tenu de ses effets pervers sur la gestion des transactions. Il faut par conséquent s'en prémunir au mieux en s'appuyant

\footnotetext{
${ }^{1}$ L'un des cas d'école illustrant ce point est celui de Senoble, à l'origine une modeste PME spécialisée dans la production de fromages de bas de gamme, mais occupant aujourd'hui la troisième place sur le marché français des produits frais. L'entreprise a conclu, dès 1984, un «contrat d'industrie » (en fait, de coopération) de trois ans avec les Centres Distributeurs E. Leclerc, tout en cherchant (et réussissant!) dans le même temps à être référencée par la plupart des autres enseignes de la distribution alimentaire française, notamment sous marque de distributeur.
} 
sur des mécanismes de contrôle efficaces. Nous pouvons les regrouper en deux catégories distinctes : les mécanismes de contrôle formels, coûteux et d'une efficacité douteuse dans le cadre de contrats incomplets, et les «normes relationnelles» projetant les relations interorganisationnelles dans la durée, seule manière de protéger les agents économiques contre toute déviance comportementale sur le long terme. Les mécanismes formels et informels de lutte contre l'opportunisme peuvent évidemment être vus comme complémentaires plutôt qu'irrémédiablement opposés (L. Poppo et T. Zenger, 2002).

\subsection{Des mécanismes de contrôle formels}

Une multiplicité de champs de recherche place l'opportunisme au cœur de leur analyse parce qu'il provoquerait indubitablement des effets négatifs ou contre-productifs en termes de performance économique et/ou organisationnelle ${ }^{2}$. De ce point de vue, si l'opportunisme est supposé avoir des effets négatifs, notamment parce qu'il ne permet pas de maximiser les profits joints d'une action commune à plusieurs partenaires (P. Joskow, 1990), on comprend que certains d'entre eux pouvant le subir cherchent à en limiter la portée, le supprimer définitivement étant sans doute une vue de l'esprit. Pour y parvenir, deux perspectives complémentaires sont alors envisageables :

- dans la lignée des travaux de O. Williamson (1975, 1985), la logique est principalement celle d'un contrôle de l'opportunisme pré et post-contractuel. Ce contrôle peut d'ailleurs être à la fois incitatif, en expliquant clairement la nature des arrangements contractuels et des sanctions appliquées aux éventuels partenaires qui s’y soustrairaient (P. Rubin, 1990), mais aussi directif, en éliminant sans pitié des partenaires jugés déloyaux ;

- dans la lignée des travaux de W. Ouchi (1980), en revanche, il est nécessaire de formaliser des procédures aboutissant à des échanges équitables, du moins perçus comme tels par les agents économiques. Il s'agit d'une action stratégique commune reposant sur l'hypothèse selon laquelle si chacun participe finalement au projet commun, c'est qu'il est convaincu de gagner plus que s'il n'y participait pas, ce qui devrait décourager toute attitude opportuniste potentielle.

\footnotetext{
${ }^{2}$ L'article de A. Rindfleisch et J. Heide (1997) propose une excellente synthèse de la littérature sur le sujet.
} 
Quelle que soit la perspective retenue, l'approche néo-institutionnelle préconise la mise en place de mécanismes de contrôle formels et très coûteux de l'opportunisme. Par exemple, il s'agira du recours systématique à des experts pour s'assurer du respect des clauses contractuelles originellement convenues, dans le but d'éviter que certains partenaires captent, grâce à leur conduite déloyale, une partie indue de la valeur créée (ou de la rente). En résumé, les mécanismes de contrôle traditionnellement évoqués dans la littérature sont, d'un côté, la qualification, avec la mise en œuvre d'un processus efficace d'évaluation et de sélection des partenaires et, de l'autre, le pilotage et la surveillance des actions conduites par les partenaires après leur sélection. Ces mécanismes se complètent d'ailleurs plus qu'ils ne s'opposent (R. Stump et J. Heide, 1996), mais ils ont pour limite principale de minimiser l'importance d'une voie alternative : la mise en place de normes relationnelles.

\subsection{La mise en place de normes relationnelles}

Force est de reconnaître que l'approche néo-institutionnelle de la firme, privilégiant des modes de contrôle formels, suscite à ce niveau de nombreuses critiques. La plus significative concerne son caractère sous-socialisé dans la mesure où elle ignore à la fois la diversité des motivations humaines en dehors du modèle «canonique» de l'homo aconomicus (M. Moschandreas, 1997) et l'importance des processus d'encastrement social entre individus et/ou entre entreprises (M. Granovetter, 1973) ${ }^{3}$. En effet, l'encastrement social dans une multiplicité de réseaux personnels, souvent sur un territoire géographique confiné, limiterait la diffusion et la multiplication de comportements déviants en jouant sur des effets de réputation, mais aussi d'intimité et d'intensité émotionnelle, dont l'importance est bien connue dans le monde des affaires, même si la réelle influence de la confiance interpersonnelle comme mécanisme efficace de contrôle de l'opportunisme prête à débat (S. Jap et E. Anderson, 2003). D'ailleurs, R. Coase (2000) lui-même défend l'idée que les effets de

\footnotetext{
${ }^{3}$ L'opposition entre O. Williamson $(1975,1985)$ et W. Ouchi (1980) symbolise à nos yeux ce dualisme, soulignant la difficile intégration par les approches économiques des apports de la sociologie des organisations. En raisonnant selon une logique de contrôle des comportements et d'émergence rationnelle de règles de bonne conduite, les approches économiques s'inscrivant dans la mouvance de O. Williamson $(1975,1985)$ font preuve d'une évidente sous-socialisation, pour reprendre M. Granovetter (1973).
} 
réputation peuvent limiter les risques d'opportunisme sans qu'il soit nécessaire de recourir à des modes d'organisation très formels.

La présence de processus d'encastrement explique que K. Provan (1993) insiste sur l'importance de la mise en place de normes relationnelles fondées sur l'équité, la transparence et la réciprocité en vue de limiter l'opportunisme, y compris en présence d'actifs hautement spécifiques et d'un faible nombre de parties prenantes. Pour ce faire, il est nécessaire d'inscrire l'échange dans la durée, en valorisant des effets de réputation (loyauté vs opportunisme) qui émergent des interactions continues entre agents économiques. L'économie des contrats, et notamment des contrats de long terme, aboutit à une conclusion similaire puisque, comme l'écrit P. Joskow (1999, p. 282), «un contrat de long terme, précisant ex ante les termes et conditions de réalisation d'un ensemble de transactions futures, donne la possibilité de se protéger ex post contre des problèmes de performance ». Il devient alors envisageable pour les partenaires de procéder à des ajustements mutuels et de trouver ensemble une solution harmonieuse aux éventuels conflits d'intérêt grâce à un « dialogue constructif» (J. Hibbard et al., 2001), dont l'intérêt majeur est de neutraliser en partie les comportements opportunistes (R. Achrol et G. Gundlach, 1999).

En conséquence, l'idée d'un dialogue constructif en vue de créer un climat apte à déjouer l'opportunisme dans le cadre d'un contrat de long terme constitue un thème d'investigation stimulant. En effet, un agent économique qui construit et tente de faire vivre une relation d'échange durable, en engageant d'importantes ressources, en escompte des partenaires plus loyaux. Il s'engage pour cela dans un travail de motivation continue pour les amener à agir dans un sens favorable aux intérêts du RIO (P. Bogetoft et H. Olesen, 2004). Si les partenaires manifestent malgré tout, de manière ponctuelle, une attitude opportuniste en violant l'une des clauses du contrat, des ajustements comportementaux seront toujours possibles, alors que l'on pourrait penser que l'opportunisme est par nature une action destructive pour les relations d'échange (J. Hibbard et al., 2001). Ceci ouvre une brèche théorique de première importance dans l'approche orthodoxe. 


\section{L'approche «hétérodoxe» ou de l'utilité des comportements opportunistes}

Faut-il vraiment chercher à se prémunir contre tout opportunisme latent ou révélé ? Au contraire, un niveau modéré ne serait-il pas tolérable, voire souhaitable? S. Dutta et al. (1994) ont émis cette idée surprenante au milieu des années 1990, sans qu'elle donne suite à des travaux qui tentent de l'approfondir. Éliminer l'opportunisme, signalent ces auteurs, si tant est qu'on puisse y parvenir, génère des coûts de gouvernance, en particulier au niveau de la surveillance ex post des comportements. Il est par conséquent légitime de s'interroger sur les gains réels que les co-contractants retirent finalement de la relation d'échange si des ressources significatives sont consacrées à des activités de surveillance. En outre, comme le signalent S. Ghoshal et P. Moran (1996) dans leur controverse avec O. Williamson (1985, 1996), plus les mécanismes de contrôle (surveillance) sont perçus comme lourds et contraignants par l'agent économique qui les subit, plus il est tenté d'être opportuniste lorsque la violation des clauses contractuelles lui semble possible sans être démasqué.

Tolérer un relatif opportunisme dans les relations entre partenaires au sein d'un RIO pourrait alors réduire de manière sensible les coûts de gouvernance et constituer une solution satisfaisante, bien que non optimale, pour l'agent économique qui les supporte. Après tout, soulignent N. Foss et C. Koch (1996), beaucoup d'observateurs oublient que la solution alternative, à savoir la construction d'une confiance entre des partenaires commerciaux, exige des investissements matériels et immatériels importants sans totalement écarter la recherche de l'intérêt personnel.

\subsection{Quelle relation entre opportunisme et confiance?}

Sans entrer dans le débat sur la nécessité ou la non-nécessité d'une confiance préalable entre co-contractants pour construire une relation de long terme et sur son rôle clé dans l'élimination de l'opportunisme, il semble difficile de totalement l'éluder. Depuis la contribution originelle de P. Ring et A. Van de Ven (1992), il est en effet entendu que la confiance se fonde à la fois sur une prévisibilité des comportements du partenaire et sur son 
niveau anticipé de bienveillance, c'est-à-dire sur sa bonne volonté à apporter une réponse satisfaisante à ce qui lui est demandé sans user de tromperie ou de mensonge. Au fur et à mesure que les différents co-contractants appartenant à un même système interorganisationnel découvrent qu'ils font preuve de loyauté et de bienveillance mutuelles, un climat de confiance s'instaure pas à pas, qui rend inutile de coûteux (et complexes) mécanismes de contrôle d'un opportunisme ayant fini par disparaître. En quelque sorte, des «jeux répétés » ont convaincu les parties en présence de la réalité de la logique coopérative (R. Axelrod, 1984).

Pourtant, dans un article peu connu, A. Van de Ven (1996) lui-même est revenu sur sa position en reconnaissant l'existence, non pas d'une courbe décroissante liant opportunisme et confiance, mais plutôt d'une courbe en U comme nous l'illustrons à l'aide de la figure 1. Son raisonnement est le suivant. Dans un premier temps, en situation de confiance grandissante entre les co-contractants, l'opportunisme se réduit progressivement, notamment grâce à une réputation fondée sur la présence de comportements loyaux clairement observables par toutes les parties; les co-contractants font la preuve continue de leur bienveillance et il devient possible d'anticiper sans difficulté majeure leurs comportements. Mais, dans un second temps, en situation de forte confiance, la tentation opportuniste risque de se manifester à nouveau car la disparition progressive des mécanismes de contrôle ouvre la voie à une ruse ou à une tromperie désormais difficilement observables. N'oublions pas, signale S. Plociniczak (2003, pp. 34-35), que «la confiance est aussi l'ingrédient qui nourrit les comportements déviants et favorise même parfois les actions délictueuses, la trahison, en d'autres termes l'opportunisme, car plus la confiance est forte, plus le gain que l'on peut espérer est élevé».

Figure 1. La relation entre opportunisme et confiance : une courbe en U

[placer ici la figure 1] 


\subsection{Vers la reconnaissance d'un niveau modéré d'opportunisme}

Si l'on couple la démarche de S. Dutta et al. (1994) à celle de A. Van de Ven (1996), nous sommes en droit de nous interroger sur la possibilité et l'intérêt d'anticiper et de sanctionner toutes les violations possibles d'un contrat ou d'une norme relationnelle fondée sur la confiance $^{4}$. Tout au plus, peut-on rester en état d'alerte en évitant que ces violations soient trop flagrantes... L'éventualité de comportements opportunistes, voire une simple attitude opportuniste, apparaissent comme une sorte d'aiguillon qui oblige les agents économiques impliqués dans un même système inter-organisationnel à rester vigilants, à éviter de tomber dans une sorte de «sclérose » ou d'inertie organisationnelle. L'avantage est évidemment de réduire les coûts de gouvernance pour l'agent économique qui craint de subir des comportements opportunistes. C'est en cela que S. Dutta et al. (1994) parlent de niveau tolérable d'opportunisme.

Pour mettre l'accent sur la dimension volontariste de ces comportements, nous retiendrons l'idée de niveau modéré d'opportunisme illustrée par l'étude d'un RIO au sens que lui donnent R. Miles et C. Snow (1986, 1995), repris par G. Paché et C. Paraponaris (2006), à savoir une combinaison d'acteurs juridiquement et financièrement indépendants aux compétences complémentaires ( $\mathrm{R} \& \mathrm{D}$, production, distribution, etc.) dont l'objectif est de mener à bien un projet commun. On peut alors se demander si l'efficacité du RIO ne peut pas être améliorée lorsque l'un des membres fait ponctuellement «cavalier seul » de manière déloyale, c'est-à-dire en opposition directe avec une clause du contrat de coopération spécifiant une obligation de transparence (par exemple, conquérir secrètement de nouveaux marchés à l'étranger qui intéressent les autres membres du RIO).

Si ledit contrat indique que toute action stratégique doit faire l'objet d'un processus collégial de prise de décision pour optimiser l'allocation de ressources, nous sommes manifestement en situation d'opportunisme actif puisque l'action du «cavalier seul » est menée volontairement

\footnotetext{
${ }^{4}$ X. Lecocq (2004) note par ailleurs que l'opportunisme au sein d'un RIO revêt un caractère éminemment subjectif: une tricherie pourra être jugée comme inacceptable par les uns, mais dans «l'ordre des choses » par les autres. Tout dépend in fine de la norme relationnelle intériorisée par chacun des partenaires. Pour l'auteur, conclure à l'existence de comportements définitivement opportunistes, c'est ainsi reconnaître qu'ils enfreignent «les normes intériorisées par les acteurs et non pas (...) des normes (quelles soient formelles ou informelles) définies collectivement $\gg$ (X. Lecocq, 2004, p. 118).
} 
en violation du contrat. Toutefois, en agissant ainsi, l'opportuniste ne va-t-il pas pouvoir assimiler de nouvelles compétences managériales, logistiques et/ou techniques qui, en retour, bénéficieront à l'ensemble des membres du RIO (meilleure connaissance de consommateurs étrangers, mise au point d'une logistique sur mesure, etc.) ? En d'autres termes, un opportunisme de court terme ne peut-il pas être utile aux membres du RIO dès lors qu'il n'obère pas le long terme et, en particulier, l'avenir du contrat formalisant le projet commun ? La performance associée au comportement déloyal concernerait dès l'instant non seulement le partenaire opportuniste, mais aussi ceux qui, a priori, en sont les victimes ${ }^{5}$.

\section{L'opportunisme modéré : un cadre d'analyse}

De la revue de la littérature, il ressort que les acteurs d'un RIO ont conscience qu'un ou plusieurs d'entre eux n'hésiteront pas à faire preuve d'opportunisme en violant le contrat de coopération dès lors que les circonstances le permettent. Une telle violation découle de l'incomplétude du contrat ou du fait que ses clauses ne peuvent pas être appliquées de manière suffisamment dissuasive pour convaincre de l'inutilité des comportements opportunistes. Mais il est également clair, sans que cela soit formalisé, que l'existence d'une menace opportuniste permet de combattre la «sclérose organisationnelle » et la bureaucratisation rampante, voire d'ouvrir pour l'ensemble du RIO des perspectives de développement insoupçonnées.

Une certaine dose d'opportunisme pourrait ainsi offrir des avantages à un double niveau. Le premier niveau, qualifié de «statique», résulte du fait que chacun des membres d'un RIO apprend à rester sur ses gardes face aux autres parties prenantes et maintient intactes ses capacités d'adaptation (et d'éventuelle sortie du RIO). Le second niveau, qualifié de «dynamique», se fonde sur des effets d'apprentissage et un processus continu

\footnotetext{
${ }^{5}$ Sans entrer en détail dans l'analyse des travaux conduits en contrôle de gestiọn sụr la mesure de la performance, notons simplement qu'il est possible de mobiliser pour cela une batterie d'indicateurs à la fois financiers et non financiers, quantitatifs et qualitatifs, etc. (D. Bessire, 1999). Il serait ainsi possible d'évaluer l'amélioration de la performance découlant du comportement opportuniste d'un membre du RIO sur un autre membre non-opportuniste du même RIO en référence à des indicateurs tels que la croissance du chiffre d'affaires, le management des ressources humaines, la qualité et la flexibilité des processus, la productivité, la capacité d'innovation, etc.
} 
d'identification des nouvelles opportunités de développement pour l'ensemble du RIO, rendues possibles par les activités d'un membre opportuniste. En bref, l'opportunisme permet peut-être de saisir des opportunités, non pas comme simple fruit du hasard, mais comme mode de création de valeur alternatif aux stratégies fondées sur la confiance. Ceci nous permet d'émettre une proposition de recherche dans la continuité de l'approche hétérodoxe, dont la discussion ne sera possible que grâce à la formalisation d'une grille de lecture des situations-types d'opportunisme.

\subsection{Proposition de recherche}

Et si un niveau modéré d'opportunisme s'avérait a priori souhaitable dans le déroulement de relations coopératives inter-organisationnelles? En formulant de la sorte notre proposition de recherche, nous poursuivons certes la perspective envisagée par S. Dutta et al. (1994), mais en adoptant l'idée d'un développement volontariste en trois étapes : d'abord «interdit», l'opportunisme va être «toléré » pour finalement être « souhaité » par les partenaires, mais à un niveau modéré. En deçà de ce niveau, un trop faible opportunisme ne constitue pas une menace crédible et conduit les agents économiques à un contentement de soi et de la situation actuelle. À l'opposé, un trop fort opportunisme risque d'entraîner des effets destructeurs irréversibles, en partie dus à des coûts de gouvernance jugés insupportables qui conduisent inéluctablement à une politique d'internalisation. Toute la question pour les preneurs de décision sera d'anticiper et de gérer le niveau souhaitable d'opportunisme, notamment en référence à un processus d'apprentissage organisationnel fondé sur la mémorisation des modes de pilotage du RIO, avant et après les manifestations de l'opportunisme.

Notre proposition de recherche s'inscrit dans la filiation de travaux anciens, mais toujours d'actualité, de L. Stern et J. Heskett (1969) sur la gestion des conflits dans les systèmes interorganisationnels de type canal de distribution. Ces travaux sont largement adaptables au cas des RIO. Si les auteurs conviennent qu'un niveau de conflit excessif est destructeur de ressources et d'énergie dans un canal de distribution, ils notent aussi qu'un niveau de conflit insuffisant risque de générer une «passivité » peu propice aux innovations techniques et managériales. Ceci pourrait encourager certains membres d'un système peu conflictuel à le 
quitter car des gains plus importants pourraient (conditionnellement) être escomptés ailleurs. Repérer le niveau souhaitable de conflit inter-organisationnel, ni trop fort ni trop faible, devient dès lors une démarche essentielle pour assurer une certaine dynamique d'évolution et de performance du canal de distribution.

Il paraît essentiel de souligner ici que l'idée d'opportunisme souhaitable, à un niveau modéré, ne remet pas en cause l'existence de normes relationnelles intériorisées par les acteurs, mais doit les conduire à rester suffisamment sur leurs gardes pour s'adapter aux évolutions de l'environnement du RIO. L. Markoczy (2003) note d'ailleurs que la plupart des agents économiques faisant preuve de vigilance, par une observation attentive et continue du comportement des autres agents, ne perdent pas automatiquement la confiance qu'ils ont placée en eux en cas de non-respect ponctuel d'un engagement. La confiance sera en revanche définitivement altérée si l'opportunisme se systématise et détruit durablement de la valeur pour celui qui le subit. En bref, plutôt que de rester passif, mieux vaut qu'un membre d'un RIO fasse preuve d'opportunisme modéré en contournant par exemple un accord d'exclusivité, ce qui permettra peut-être d'offrir des «opportunités » de croissance pour les autres membres. Afin d'identifier les formes d'opportunisme et de disposer ainsi d'un outil original d'analyse, nous proposons ici une grille de lecture synthétisant les approches orthodoxe et hétérodoxe, sur laquelle nous greffons trois indicateurs de l'opportunisme dans un RIO issus des travaux de A. Lapointe et S. Pageau (2000).

\subsection{RIO et situations-types d'opportunisme, une grille de lecture}

La manière dont les comportements opportunistes sont envisagés par les approches orthodoxe et hétérodoxe varie finalement selon deux axes principaux. Le premier axe s'appuie sur l'acceptation ou le refus de ces comportements par les membres d'un RIO; le second axe se réfère à la dimension temporelle dans laquelle se situent les membres d'un RIO, en distinguant l'horizon de court terme de l'horizon de long terme. Le tableau 1 fait ainsi ressortir quatre situations-types synthétisant les apports de la littérature sur le thème. Ces quatre situations-types permettent de disposer d'une grille de lecture à visée opératoire pour conduire un audit organisationnel des manifestations de l'opportunisme dans le cadre d'un 
RIO, en expliquant en quoi elles auront des effets positifs ou négatifs sur la performance des partenaires.

Tableau 1. Opportunisme dans un RIO : les quatre situations-types

$$
\text { [placer ici le Tableau 1] }
$$

Selon l'approche orthodoxe, éviter la désagrégation du RIO conduit à mettre en place des mécanismes de contrôle de plus en plus fins, soit de nature coercitive, en risquant de diminuer les bénéfices retirés de comportements opportunistes, soit de nature non coercitive, en récompensant les comportements loyaux par le biais de stimulants adaptés (K. Wathne et J. Heide, 2000). Cependant, au fil du temps, ces mécanismes finissent par devenir tellement coûteux que les membres du RIO reconnaissent petit à petit le caractère inéluctable de son existence, d'autant que par le biais de «dialogues constructifs » (J. Hibbard et al., 2001), il sera sans doute possible d'en limiter les effets les plus destructeurs. Les membres d'un RIO n'acceptent pas, par principe, l'idée que des comportements opportunistes puissent se propager; ils admettent toutefois qu'il s'agit de la manifestation du fait que tout agent économique poursuit et poursuivra toujours son propre intérêt (cas $n^{\circ} 1$ et cas $n^{\circ} 2$ du tableau 1).

Si nous nous plaçons maintenant dans la perspective de l'approche hétérodoxe, nous sommes en présence de comportements déviants soupçonnés par chacun des co-contractants qui, plutôt que de renoncer à la coopération (et ses bénéfices attendus), en tolèrent l'existence, jusqu'à leur reconnaître de possibles avantages pour les acteurs non-opportunistes. La violation du contrat fait partie de l'univers des possibles. Chacun s'attend à ce que l'autre enfreigne une règle en donnant l'illusion de la respecter ou trahisse une solidarité réticulaire construite pourtant depuis plusieurs années. Cela est certes jugé par nature déloyal, mais finalement nonentropique pour le RIO, voire potentiellement productif pour ses membres, à la condition évidente que l'opportunisme révélé ne menace pas leur pérennité (cas $n^{\circ} 4$ du tableau 1), et ne se manifeste que par des actions ponctuelles sur le court terme (cas $\mathrm{n}^{\circ} 3$ du tableau 1$)$. 
La constitution de la grille de lecture proposée par le tableau 1 est une première étape dans la formalisation de l'outil d'audit organisationnel évoqué précédemment. La seconde étape nécessite de recourir à des indicateurs permettant de qualifier les différentes facettes envisageables de l'opportunisme dans un RIO. Pour cela, nous nous appuyons sur la contribution de A. Lapointe et S. Pageau (2000). Se fondant sur une étude de cas de trois RIO au Québec, l'un dans le secteur de la santé (quatre ans d'existence), l'autre dans le secteur du mobilier (dix ans d'existence), et le dernier dans l'ingénierie d'automation (cinq ans d'existence), les auteurs en tirent la conclusion que l'opportunisme peut revêtir finalement trois visages différents : la tricherie, la trahison et la temporisation (voir tableau 2).

Tableau 2. Visages de l'opportunisme dans un RIO

(adapté de A. Lapointe et S. Pageau, 2000, p. 8)

[placer ici le Tableau 2]

La trahison et la tricherie sont les visages les plus connus dans la littérature, car ils renvoient directement à la vision développée par O. Williamson $(1985,1993)$ : cesser d'être fidèle à un partenaire ou enfreindre la clause d'un contrat, avec pour objectif dans les deux cas d'en tirer un bénéfice personnel, consiste bien à agir avec ruse au détriment de l'autre partie. En revanche, il n'est pas sûr que la temporisation puisse être totalement assimilée à un véritable comportement opportuniste. Il peut s'agir simplement de l'expression d'une rationalité économique qui conduit tout agent à essayer de différer le plus possible une décision par nature risquée pour lui. La contribution de A. Lapointe et S. Pageau (2000) débouche sur des catégories «actionnables » dans le cadre d'une recherche de terrain. En effet, à partir du tableau 2, il est possible d'interpréter un ensemble d'actions stratégiques comme relevant de comportements de nature opportuniste, pour ensuite analyser si cet opportunisme aura un effet positif ou négatif sur la performance des membres du RIO. Les parties prenantes du projet commun pourront dès lors s'interroger sur l'intérêt ou non d'y maintenir leur participation, évidemment en fonction des coûts irrécouvrables à supporter. 


\section{Conclusion}

Les publications académiques sur les RIO affirment quasi-unanimement que l'objectif poursuivi par les acteurs est de se prémunir contre les conduites opportunistes des «partenaires-adversaires » (H. Chesbrough et D. Teece, 1996 ; G. Konig, 1996 ; C. Jameux, 1998). En ce sens, une certaine orthodoxie de la recherche en théorie des organisations et en management stratégique considère l'opportunisme comme néfaste pour les relations interorganisationnelles; pour éviter qu'il annule les effets attendus d'une coopération, les acteurs sont alors encouragés à se doter de mécanismes de contrôle et de sanction appropriés. Une telle conception est cependant remise en cause par un ensemble de travaux plus hétérodoxes qui estiment, au contraire, que l'opportunisme peut renforcer la compétitivité au sein des RIO. À partir d'une analyse de la littérature, aboutissant à proposer une grille de lecture à visée opératoire pour réaliser un audit organisationnel des manifestations de l'opportunisme, notre réflexion s'inscrit dans cette vision en considérant qu'il y existe un niveau souhaitable d'opportunisme.

Il conviendrait, à partir du cadre d'analyse proposé, de mener des investigations de terrain, tout particulièrement dans les domaines où des évolutions récentes permettent à des entreprises industrielles et commerciales de coopérer plus aisément tout en optant dans le même temps pour des stratégies prédatrices et des comportements opportunistes. C'est notamment le cas des technologies de l'information et de la communication (TIC), avec le développement de puissants systèmes d'information inter-organisationnels (SIIO) et le recours grandissant à des places de marché électroniques (PdMÉ) qui associent étroitement logiques de coopération (partenariats logistiques) et logiques de compétition (processus d'enchères inversées). À n'en point douter, il s'agit d'une voie de recherche des plus prometteuses sur la dynamique d'évolution des relations inter-organisationnelles pour le management stratégique. 


\section{Bibliographie}

Achrol, R., Gundlach, G. Legal and social safeguards against opportunism in exchange. Journal of Retailing, 1999, vol. 75, $\mathrm{n}^{\circ}$ 1, p. 107-124.

Axelrod, R. The evolution of cooperation. New York, NY : Basic Books, 1984.

Barreyre, P.-Y. L'impartition : politique pour une entreprise compétitive, Paris : Hachette, 1968.

Baudry, B. L'économie des relations interentreprises, Paris : La Découverte, 2006, $\mathrm{n}^{\text {Ile }}$ éd.

Bessire, D. Définir la performance. Comptabilité Contrôle Audit, 1999, vol. 5, n 2, p. 127-150.

Bogetoft, P., Olesen, H. Design of production contracts: lessons from theory and agriculture, Copenhagen : Copenhagen Business School Press, 2004.

Brown, J., Dev, C., Lee, D.-J. Managing marketing channel opportunism : the efficacy of alternative governance mechanisms. Journal of Marketing, 2000, vol. 64, $\mathrm{n}^{\circ}$ 2, p. 51-65.

Capraro, M., Baglin, G. L'entreprise étendue et le développement des fournisseurs, Lyon : Presses Universitaires de Lyon, 2002.

Chalmin, P. Tate and Lyle, géant du sucre, Paris : Economica, 1983.

Chesbrough, H., Teece, D. When is virtual virtuous ? Organizing for innovation. Harvard Business Review, 1996, vol. 74, $\mathrm{n}^{\circ}$ 1, p. 65-74.

Coase, R. L'économie néo-institutionnelle. Revue d'Économie Industrielle, 2000, n 92, p. 51-54.

Crozier, M., Friedberg, E. L'acteur et le système. Les contraintes de l'action collective. Paris : Éditions du Seuil, 1977.

Dutta, S., Bergen, M., John, G. The governance of exclusive territories when dealers can bootleg. Marketing Science, 1994, vol. 13, n 1, p. 83-99.

Foss, N., Koch, C. Opportunism, organizational economics and the network approach. Scandinavian Journal of Management, 1996, vol. 12, n 2, p. 189-205.

Ghoshal, S., Moran, P. Bad for practice: a critique of the transaction cost theory. Academy of Management Review, 1996, vol. 21, n 1, p. 13-47.

Granovetter, M. The strength of weak ties. American Journal of Sociology, 1973, vol. 78, $\mathrm{n}^{\circ}$ 6, p. $1360-1380$.

Hart, O., Holmstrom, B. The theory of contracts. In Bewley, T. (Ed.), Advances in economic theory. Cambridge, MA : Cambridge University Press, 1987, p. 305-329.

Hibbard, J., Kumar, N., Stern, L. (2001), Examining the impact of destructive acts in marketing channel relationships. Journal of Marketing Research, 2001, vol. 38, ${ }^{\circ}$ 1, p. 45-61.

Jameux, C. Pouvoir et confiance : retour sur la nature et le rôle de l'autorité dans le fonctionnement des organisations. Économies et Sociétés, Série Sciences de Gestion, 1998, nº 8-9, p. 87-98.

Jameux, C. D'un modèle de référence à une classe de problèmes récurrents en gestion. In Voisin, C., Ben Mahmoud-Jouini, S., Edouard, S. (Éds.), Les réseaux: dimensions stratégiques et organisationnelles. Paris : Economica, 2004, p. 45-57.

Jap, S., Anderson, E. Safeguarding interorganizational performance and continuity under ex post opportunism. Management Science, 2003, vol. 49, $\mathrm{n}^{\circ} 12$, p. 1684-1701.

Joffre, P. L'économie des coûts de transaction ou le marché et l'entreprise à la fin du XXe siècle. In Kœnig, G. (Éd.), De nouvelles théories pour gérer l'entreprise du XXI ${ }^{\mathrm{e}}$ siècle. Paris : Economica, 1999, p. 143-170.

Joshi, A., Stump, R. Determinants of commitment and opportunism : integrating and extending insights from transaction cost analysis and relational exchange theory. Revue Canadienne des Sciences de l'Administration, 1999, vol. 16, n 4, p. 334-352.

Joskow, P. Vertical integration and long-term contracts : the case of coal-burning electric generating plants. In Williamson, O. (Ed.), Industrial organization. Cheltenham: Edward Elgar, 1990, p. 281328. 
Joskow, P. Contract duration and relationship-specific investments : empirical evidence from coal markets. In Williamson, O., Masten, S. (Eds.), The economics of transaction costs. Cheltenham: Edward Elgar, 1999, p. 281-298.

Julien, P.-A., Raymond, L., Jacob, R., Abdul-Nour, G. Introduction. In Julien, P.-A., Raymond, L., Jacob, R., Abdul-Nour, G. (Éds.), L'entreprise-réseau. Dix ans d'expérience de la Chaire Bombardier Produits récréatifs. Sainte-Foy : Presses de l’Université du Québec, 2003, p. 1-43.

Kœnig, G. Management stratégique. Paradoxes, interactions et apprentissages. Paris : Nathan, 1996.

Lapointe, A., Pageau, S. Les réseaux d'entreprises: saisir l'opportunité, ou être saisi d'opportunisme? Actes du Cinquième Congrès International Francophone sur la PME, Lille, octobre 2000, p. 1-17 (CD-rom).

Lecocq, X. Une approche socio-cognitive de l'opportunisme: le cas d'un réseau interorganisationnel européen. M@n@gement, 2004, vol.7, nº 3, p. 109-135.

Markoczy, L. Trust but verify : distinguishing distrust from vigilance. Unpublished Working Paper, Version 1.33, Anderson Graduate School of Management, Riverside, CA : University of California, July 2003, URL : http://www.goldmark.org/livia.

Ménard, C. The economics of hybrid organizations. Journal of Institutional and Theoretical Economics, 2004, vol. 160, n 3, p. 345-376.

Miles, R., Snow, C. Organizations : new concepts for new forms. California Management Review, 1986, vol. 28, n 3, p. 62-73.

Miles, R., Snow, C. The new network firm: a spherical structure built on a human investment philosophy. Organizational Dynamics, 1995, vol. 23, nº 4, p. 5-18.

Milgrom, P., Roberts, J. Économie, organisation et management. Grenoble-Bruxelles : PUG-De Boeck Université, 1997.

Montmorillon (de), B. Croissance de l'entreprise. In Joffre, P., Simon, Y. (Éds.), Encyclopédie de gestion. Paris : Economica, 1997, 2 éd., p. 854-877.

Moschandreas, M. The role of opportunism in transaction cost economics. Journal of Economic Issues, 1997, vol. 31, n 1, p. 39-57.

Ouchi, W. Markets, bureaucracies, and clans. Administrative Science Quarterly, 1980, vol. 25, n 2, p. 129-141.

Paché, G., Paraponaris, C. L'entreprise en réseau : approches inter et intra-organisationnelles. Les Éditions de l'ADREG, 2006, URL : http://www.asso.nordnet/adreg.

Plociniczak, S. Théorie des coûts de transaction et frontières de la firme: l'incomplétude de la logique transactionnelle. De la forme hybride aux réseaux inter-firmes. Actes du Colloque IREGE sur L'économie de la firme : quelles nouveautés ?, Annecy, avril 2003, p. 1-59 (CD-rom).

Poppo, L., Zenger, T. Do formal contracts and relational governance function as substitutes or complements? Strategic Management Journal, 2002, vol. 23, n 8, p. 707-725.

Provan, K. Embeddedness, interdependence, and opportunism in organizational supplier-buyer networks. Journal of Management, 1993, vol. 19, n 4, p. 841-856.

Quélin, B. Les frontières de la firme. Paris : Economica, 2002.

Rindfleisch, A., Heide, J. Transaction cost analysis : past, present, and future applications. Journal of Marketing, 1997, vol. 61, n 4, p. 30-54.

Ring, P., Van de Ven, A. Structuring cooperative relationships between organizations. Strategic Management Journal, 1992, vol. 13, nº 7, p. 483-498.

Rubin, P. Managing business transactions : controlling the cost of coordinating, communicating, and decision making. New York, NY : The Free Press, 1990.

Stern, L., Heskett, J. Conflict management in interorganization relations : an exploration. In Stern, L. (Ed.), Distribution channels : behavioral dimensions. Boston, MA : Houghton-Mifflin, 1969, p. 288305. 
Stump, R., Heide, J. Controlling supplier opportunism in industrial relationships. Journal of Marketing Research, 1996, vol. 33, n 4, p. 431-441.

Uzzi, B. Social structure and competition in interfirm networks: the paradox of embeddedness. Administrative Science Quarterly, 1997, vol. 42, n 1, p. 35-67.

Van de Ven, A. The evolution of trust and opportunism in interorganizational relationships. Presentation in the Symposium on "Trust vs opportunism in interorganizational collaboration" at the Academy of Management Annual Conference, Cincinnati (OH), August 1996, p. 1-6.

Wathne, K., Heide, J. Opportunism in interfirm relationships : forms, outcomes, and solutions. Journal of Marketing, 2000, vol. 64, $n^{\circ}$ 4, p. 36-51.

Williamson, O. Markets and hierarchies : analysis and antitrust implications. New York, NY: The Free Press, 1975.

Williamson, O. The economic institutions of capitalism : firms, markets, relational contracting. New York, NY : The Free Press, 1985.

Williamson, O. Opportunism and its critics. Managerial and Decision Economics, 1993, vol. 14, $\mathrm{n}^{\circ} 2$, p. 97-107.

Williamson, O. Economic organization : the case for candor. Academy of Management Review, 1996, vol. 21, n 1, p. 48-57. 
Figure 1. La relation entre opportunisme et confiance : une courbe en $U$

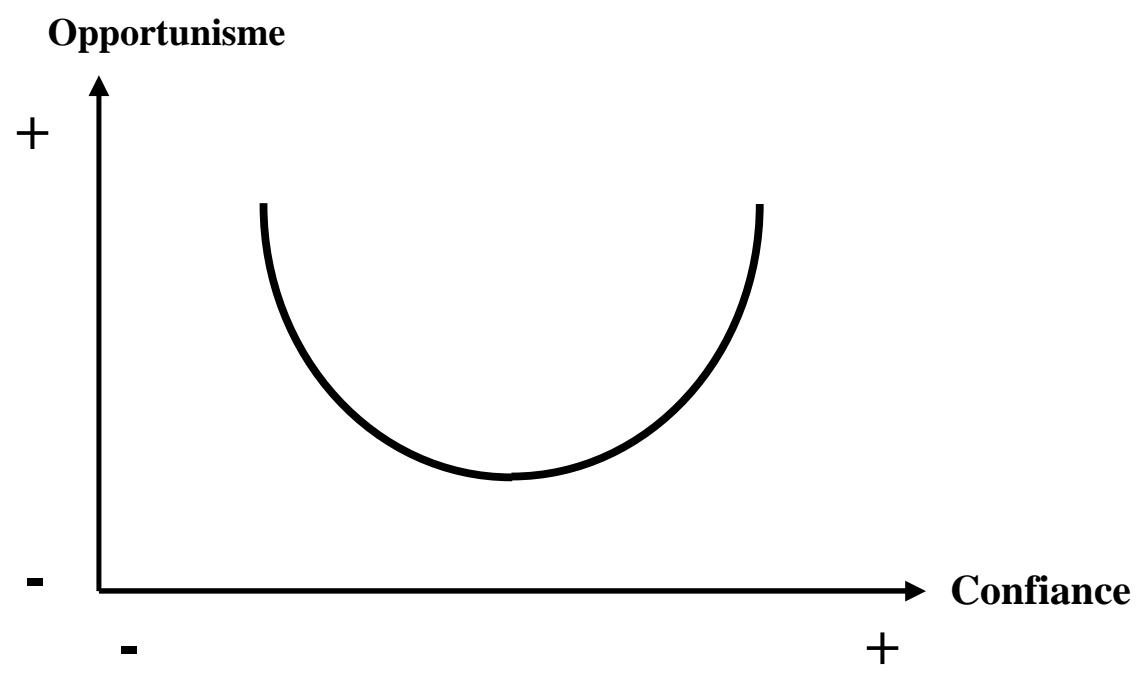


Tableau 1

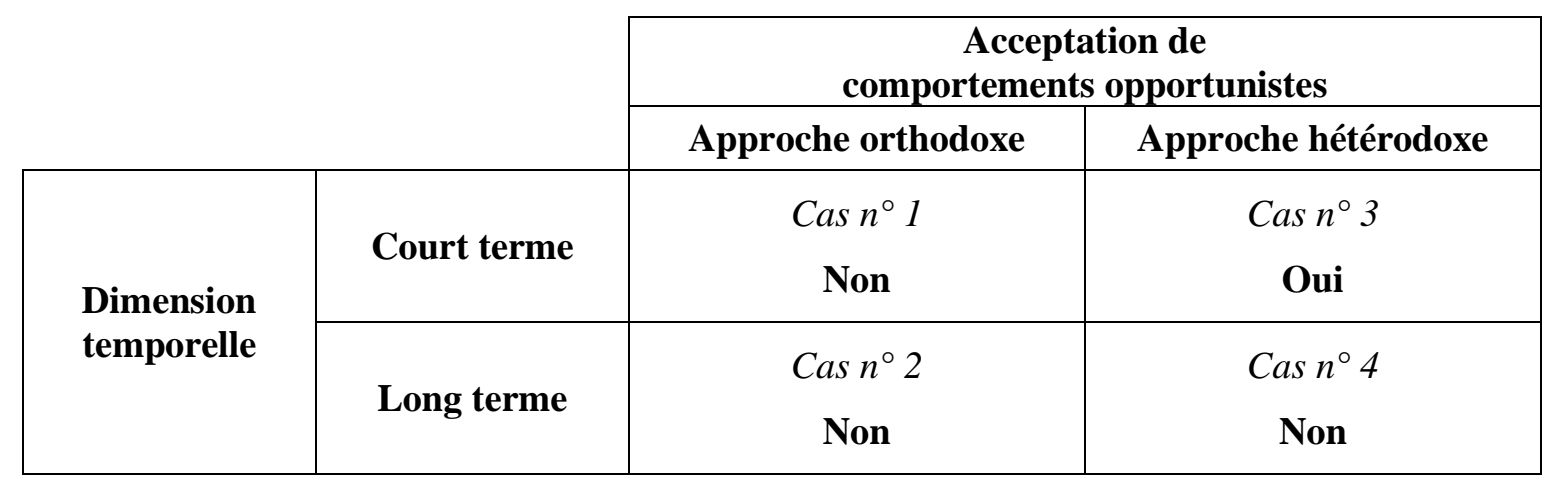




\section{Tableau 2}

\begin{tabular}{|c|c|c|}
\hline Type d'opportunisme & Définition & Exemple de manifestation \\
\hline Tricherie & $\begin{array}{c}\text { Enfreindre une clause } \\
\text { contractuelle en affectant } \\
\text { de la respecter }\end{array}$ & $\begin{array}{c}\text { Contourner secrètement } \\
\text { l'accord d'exclusivité qui lie } \\
\text { l'entreprise aux autres } \\
\text { membres du RIO }\end{array}$ \\
\hline Trahison & $\begin{array}{c}\text { Cesser d'être fidèle à un } \\
\text { co-contractant auquel on } \\
\text { est lié par une solidarité }\end{array}$ & $\begin{array}{c}\text { Mettre en ouvre le projet } \\
\text { productif élaboré en commun } \\
\text { en excluant finalement l'un } \\
\text { des membres du RIO }\end{array}$ \\
\hline Temporisation & $\begin{array}{c}\text { Différer l'action par calcul, } \\
\text { dans l'attente d'un } \\
\text { moment plus favorable }\end{array}$ & $\begin{array}{c}\text { Attendre la signature du contrat } \\
\text { garantissant des retombées à } \\
\text { court terme avant d'engager } \\
\text { des ressources dédiées }\end{array}$ \\
\hline
\end{tabular}

